\title{
Checklist dos escorpiões (Arachnida, Scorpiones) do Mato Grosso do Sul, Brasil
}

\author{
Leonardo S. Carvalho ${ }^{1,2}$, Antonio D. Brescovit³, Cláudio A. R. Souza ${ }^{3}$ \& Josué Raizer ${ }^{4}$
}

\author{
1. Universidade Federal do Piauí, Campus Amílcar Ferreira Sobral, BR 343, Km 3,5,64800-000 Floriano, Pl, Brasil. (carvalho@ufpi.edu.br) \\ 2. Programa de Pós-Graduação em Zoologia, Universidade Federal de Minas Gerais, Belo Horizonte, MG, Brasil. \\ 3. Instituto Butantan, Laboratório Especial de Coleçōes Zoológicas, Av. Vital Brasil, 1500, Butantã, 05503-900 São Paulo, SP, Brasil. \\ 4. Universidade Federal da Grande Dourados, Faculdade de Ciências Biológicas e Ambientais, Rodovia Dourados-ltahum km 12, Cidade Universitária, 79825-070
} Dourados, MS, Brasil.

Recebido 7 dezembro 2016

Aceito 6 fevereiro 2017

DOI: $10.1590 / 1678-4766 e 2017108$

\begin{abstract}
Checklist of the scorpions (Arachnida, Scorpiones) from Mato Grosso do Sul State, Brazil. In this paper it is provided a checklist of Scorpiones species in the state of Mato Grosso do Sul, Brazil. There are 16 species and one non-nominotypical subspecies recorded for the state, although it is discussed the presence and/or the taxonomic status of three of these species. This, it is considered that the scorpiofauna of the Mato Grosso do Sul is actually composed of 13 species, which represents more than $50 \%$ of all known species from Central-western Brazil. Two bothriurid species are known only for this state and are considered endemics: Bothriurus pora Mattoni \& Acosta, 2005 and Brazilobothriurus pantanalensis Lourenço \& Monod, 2000. The distribution of the scorpion registries in the Mato Grosso do Sul is heterogeneous, being the areas dominated by Atlantic Forest and Pantanal is sampled worse than those covered by the Cerrado domains.
\end{abstract}

KEYWORDS. Bothriuridae, scorpiofauna, Central-western Region, Biota sulmatogrossense, BIOTA/MS Program.

RESUMO. Nesse artigo é fornecida uma lista de espécies de Scorpiones do estado do Mato Grosso do Sul, Brasil. Até o momento 16 espécies e uma subespécie não-nominotípica estão registradas para o estado, porém é discutida a presença e/ou o status taxonômico de três destas espécies. Assim, considera-se que a escorpiofauna do Mato Grosso do Sul é composta, até o momento, por 13 espécies, o que representa mais de 50\% de todas as espécies de escorpiões conhecidas para o Centro-Oeste brasileiro. Duas espécies de bothriurídeos são conhecidas apenas para este estado e consideradas endêmicas: Bothriurus pora Mattoni \& Acosta, 2005 e Brazilobothriurus pantanalensis Lourenço \& Monod, 2000. A distribuição dos registros de escorpiões no Mato Grosso do Sul é heterogênea, uma vez que as áreas dominadas por Mata Atlântica e pelo Pantanal são menos amostradas em relação àquelas de domínios do Cerrado.

PALAVRAS-CHAVE. Bothriuridae, escorpiofauna, Região Centro-Oeste, Biota sulmatrogrossense, Programa BIOTA/MS.

Os escorpiões compõem uma ordem relativamente pequena de aracnídeos, com cerca de 1.500 espécies distribuídas em 163 gêneros e 18 famílias (Fet et al., 2000; LOURENÇO, 2002; Pinto-DA-Rocha et al., 2007; BrazIL \& PORTO, 2011). No Brasil são conhecidas 131 espécies descritas em 23 gêneros, distribuídos em apenas 4 famílias, representando cerca de $9 \%$ da diversidade mundial do grupo (BrazIL \& PorTo, 2011). Esta riqueza poderia ser considerada elevada mas encontra-se subestimada, visto a grande dimensão territorial do Brasil, aliado à variedade de ecossistemas, com ambientes áridos ou semiáridos (Cerrado e Caatinga) e úmidos (Amazônia e Floresta Atlântica) (LOURENÇO \& EICKSTEDT, 2009; PorTo et al., 2010b; BRAZIL $\&$ Porto, 2011). Por outro lado, este panorama pode ser explicado pela carência de especialistas em sistemática de escorpiões no Brasil, aliado à heterogeneidade na amostragem do grupo no país, havendo extensas áreas mal amostradas e que certamente podem apresentar diversos elementos endêmicos (LouRENÇO et al., 2004).
Tais fatores tornam-se preocupantes, visto a importância médico-epidemiológica dos escorpiões. Estes animais representam sérios problemas de saúde pública, devido à ação do seu veneno em caso de acidente. No Brasil estima-se que ocorram 8.000 acidentes/ano, com um coeficiente de incidência anual de aproximadamente 3 casos/100.000 habitantes (LIRA-DA-SILVA et al., 2009). Apenas 25 espécies de escorpiões no mundo, todas pertencentes à Buthidae, são reconhecidamente causadoras de acidentes graves (Lourenço \& Eickstedt, 2009; Brazil \& Porto, 2011). No entanto, embora a caracterização do veneno e do acidente não seja conhecida para todas as espécies de escorpiões, diversos relatos de escorpionismo têm sido reportados para espécies que ocorrem no Brasil (Álvares et al., 2006; CARVAlHo et al., 2007; AlbuQuerQue et al., 2009; BRANDÃo \& FranÇOSO, 2010; PorTo et al., 2010a).

Além destes problemas listados acima, não existem catálogos atualizados ou revisões taxonômicas extensas dos escorpiões brasileiros, de forma que para conseguir 
informações sobre a distribuição das espécies de uma determinada região, ou país, é preciso recorrer à literatura primária, além do catálogo de Fet et al. (2000). O mesmo não ocorre para aranhas, grupo para o qual se pode produzir uma lista de espécies de um país através de uma consulta ao catálogo eletrônico disponível on line (Brescovit et al., 2011). No entanto, isto não inviabiliza a produção de checklists da diversidade de escorpiões para determinadas regiões do Brasil, como apresentado por CANDIDO (1999) para São Paulo e por PorTo et al. (2010b) para a Bahia, além do trabalho monográfico sobre escorpiões do Brasil (LOURENÇO, 2002).

Objetiva-se apresentar uma atualização da lista de espécies de escorpiões do estado do Mato Grosso do Sul, localizado na Região Centro-Oeste do Brasil. A escorpiofauna do Mato Grosso do Sul é relativamente bem divulgada em trabalhos científicos, especialmente os recentes (LOURENÇO et al., 2004; Bertani et al., 2005; Mattoni \& Acosta, 2005) e apresenta algumas espécies endêmicas, tais como Brazilobothriurus pantanalensis (LouRENÇO \& MONOD, 2000). Atualmente são registradas para o estado do Mato Grosso do Sul 13 espécies de escorpiões (Brazil \& Porto, 2011).

\section{MATERIAL E MÉTODOS}

Para compor a lista de espécies foram buscados registros na literatura de escorpiões coletados e/ou descritos para o estado do Mato Grosso do Sul, tais como BerTani et al. (2005), BORELLI (1901), BÜCHERL (1960-1962), FET et al. (2000), LOURENÇO \& MONOD (2000), LOURENÇO (1979; 1982; 2000), LOURENÇO (2002), LouRENÇO et al. (2004) e Maury $(1970,1974,1997)$. Para complementar esta lista, acrescentou-se ainda os registros de escorpiões tombados nas coleções do Instituto Butantan (IBSP, curadora D. M. Battesti) e Museu de Zoologia da Universidade de São Paulo (MZSP, curador R. Pinto da Rocha), ambas do Estado de São Paulo, Brasil.

Ao realizar-se a pesquisa em literatura primária (como as descrições originais das espécies), foi possível encontrar registros de escorpiões em outras coleções científicas, que estão mencionadas na Tabela I. São elas: BMNH, The Natural History Museum, Londres, Inglaterra; CZUNB, Coleção Zoológica da Universidade de Brasília, Brasília, Brasil; FHC, Facultad de Humanidades y Ciencias, Montevideu, Uruguai; MHNG, Museum d'Histoire Naturelle de Geneve, Genebra, Suíça; MNHN, Museum National d'Histoire Naturelle, Paris, França; MNRJ, Museu Nacional do Rio de Janeiro, Rio de Janeiro, Brasil; ZMH, Zoologische Staatsinstitut, Zoologisches Museum Hamburg, Hamburgo, Alemanha.

Para a confecção do mapa de distribuição, os registros que não apresentavam coordenadas geográficas na publicação original foram georreferenciados utilizando banco de dados de coordenadas geográficas, seguindo a metodologia empregada por BRESCOVIT et al. (2011).

\section{RESULTADOS E DISCUSSÃO}

\section{Lista das espécies do Estado do Mato Grosso do}

Sul. Foram encontrados registros de 16 espécies e uma subespécie para o estado do Mato Grosso do Sul, distribuídos em cinco gêneros e duas famílias de escorpiões (Tab. I, Figs. 1A-F). Bothriuridae está representada por quatro espécies, enquanto que Buthidae por 12 espécies e uma subespécie. Dentre os bothriurídeos, Bothriurus pora e Brazilobothriurus pantanalensis (Fig. 1) são espécies endêmicas do estado do Mato Grosso do Sul e conhecidas apenas nas suas localidadestipo, respectivamente nos municípios de Ponta Porã e Corumbá. Além disto, Brachistosternus simoneae, descrita originalmente apenas para o estado de Goiás (LouRENÇO, 2000), é registrada pela primeira vez para o Mato Grosso do Sul, ampliando sua distribuição.

Dentre os butídeos, foram registradas duas espécies de Ananteris Thorell e dez de Tityus C. L. Koch. Ananteris balzanii é a espécie com a maior distribuição geográfica dentro do gênero, ocorrendo desde a Argentina, Paraguai, Bolívia até o norte e nordeste do Brasil (Lourenço, 2002; Pinto-DA-Rocha et al., 2007), habitando apenas áreas abertas de vegetação tipo savana (LOURENÇO, 2002). Ananteris mariaterezae, embora não esteja listada como presente no estado do Mato Grosso do Sul no trabalho de GiUPPONi et al. (2009), ocorre neste estado, visto que um indivíduo procedente de Corumbá, na zona do Pantanal sul matogrossense, foi utilizado na descrição original desta espécie (Lourenço, 1982). Além disto, Lourenço (2002) reafirmou a ocorrência desta espécie nesta localidade, informando ainda que as duas localidades (Corumbá-MS e Ilha do Bananal-TO) com registros desta espécie estão inseridas no bioma Pantanal.

Dos táxons pertencentes ao gênero Tityus e registrados para o Estado do Mato Grosso do Sul, alguns necessitam ser avaliados com mais detalhes. Tityus serrulatus (Fig. 1B) e $T$. bahiensis (Figs 1C, D) apresentam ampla distribuição pelo Brasil e são reconhecidas por serem causadoras de acidentes graves em seres humanos (LOURENÇO \& EICKSTEDT, 2009). Tityus paraguayensis (Fig. 1E) possui distribuição restrita ao estado do Mato Grosso do Sul (LourenÇo, 2002; BrazIL \& PORTO, 2011). Esta espécie ocorre em ambientes de vegetação aberta ou de savana, assim como o T. mattogrossensis, que ocorre desde o centro-oeste até o nordeste do Brasil (LOURENÇO, 2002; BrazIL \& PorTO, 2011). Tityus trivittatus (Fig. 1F) ocorre na Argentina, Paraguai e Brasil (Mato Grosso do Sul, Paraná e São Paulo) (Lourenço, 2002).

Tiyus lutzi possui status taxonômico que necessita ser revisto, considerando-se que não é citada em trabalhos científicos desde sua descrição original, e nem está presente em revisões recentes da escorpiofauna brasileira (LouRENÇO, 2002). Seu nome foi proposto em substituição à Tityus intermedius Lutz \& Mello, 1992, pré-ocupado por Tityus intermedius Borelli, 1901 (FET et al., 2000). Além disto, de acordo com FET et al. (2000), o holótipo foi perdido. 

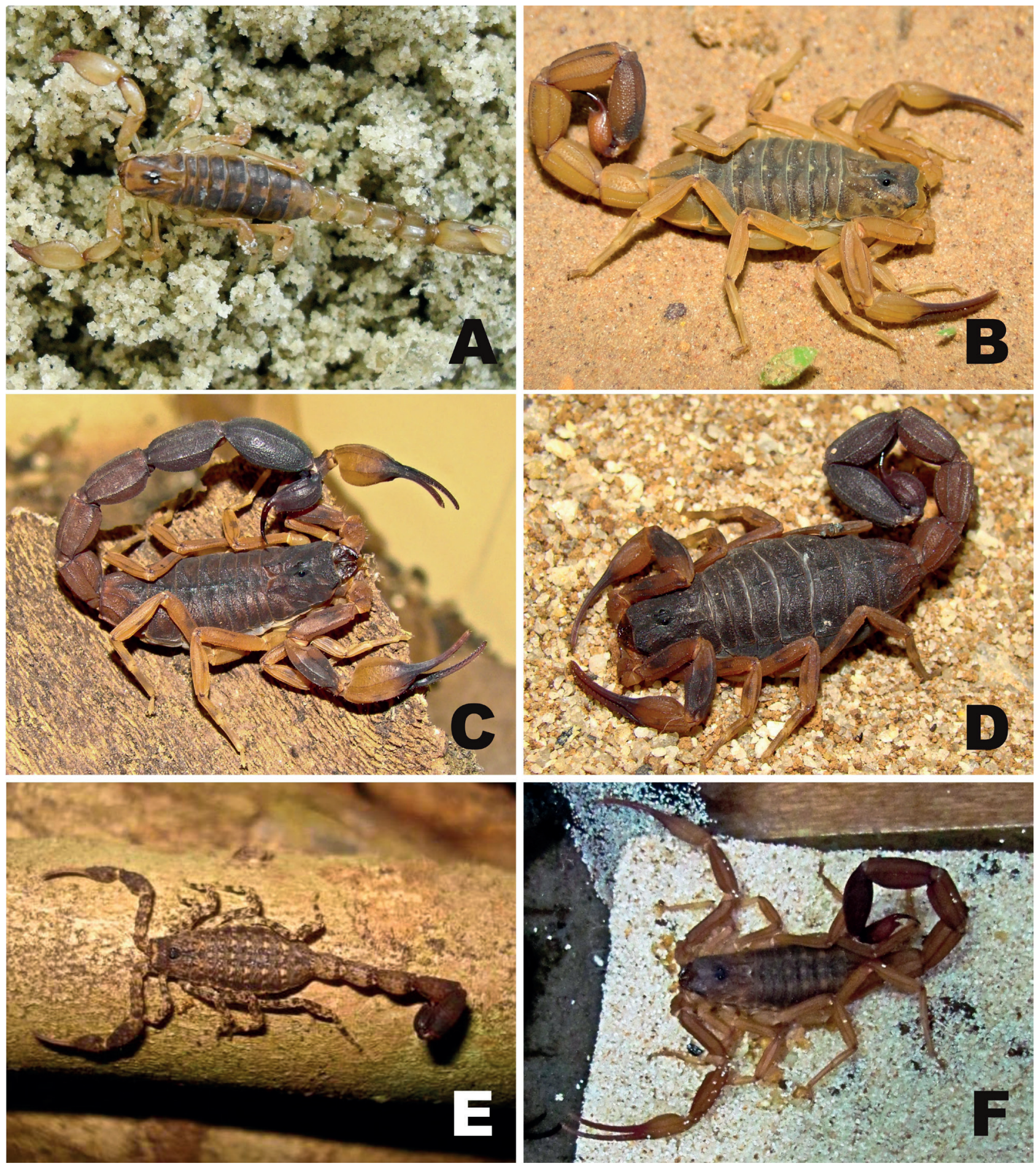

Fig. 1. Escorpiões do estado do Mato Grosso do Sul, Brasil. A), Brazilobothriurus pantanalensis; B), Tityus serrulatus, fêmea; C-D), Tityus bahiensis, macho (C) e fêmea (D); E), Tityus paraguayensis, macho; F) Tityus trivittatus, macho. Fotos de M. C. Schneider (A), L. S. Carvalho (B), R. P. Indicatti (C-D), D. Araújo (E), V. F. Mattos (F).

Situação semelhante ocorre com Tityus indecisus, que pode ser um sinônimo-júnior de T. mattogrossensis devido à sua similaridade morfológica e proximidade da localidade-tipo de ambas (Lourenço, 1979; Fet et al., 2000). Apenas com o exame do holótipo desta espécie (disponível no MNRJ, segundo NogUEIRA \& KURY, 1999), isto poderia ser solucionado.
A presença de $T$. costatus para o estado foi reportada em Brazil \& PORTO (2011), mas parece ser duvidosa. Esta distribuição diverge daquelas apresentadas por FET et al. (2000) e LOURENÇo (2002), que afirmaram que T. costatus ocorre no Espírito Santo, Minas Gerais, Rio de Janeiro, São Paulo ao Rio Grande do Sul. Nenhum registro de T.costatus 
Tab. I. Lista das espécies de Scorpiones registradas para o estado do Mato Grosso do Sul. Municípios com registros: 1, Agachi; 2, Anastácio; 3, Anaurilândia; 4, Aquidauana; 5, Bataguassu; 6, Bonito; 7, Brasilândia; 8, Campo Grande; 9, Corumbá; 10, Cuiabá; 11, Dois Irmãos do Buriti; 12, Eldorado; 13, Ilha Grande; 14, Ilha São Francisco; 15, Ivinhema; 16, Miranda; 17, Mundo Novo; 18, Paranaíba; 19, Ponta Porã; 20, Porto Murtinho; 21, Presidente Epitácio; 22, Santa Rita do Pardo; 23, Terenos; 24, Toca da Onça; 25, Três Lagoas; 26, Viral. Referências listadas: A, BerTANi et al. (2005); B, Borelli (1901); C, Brazil \& Porto (2011); D, Bücherl (1959); E, Bücherl (1960/62); F, Fet et al. (2000); G, Lourenço \& Monod (2000); H, LourenÇo (1974); I, Lourenço (1982); J, Lourenço (2000); K, Lourenço (2002); L, Lourenço et al. (2004); M, MatToni \& Acosta (2005); N, Maury (1970); O, MAURY (1979); P, Souza et al. (2009).

\begin{tabular}{|c|c|c|c|c|c|}
\hline Táxon & $\begin{array}{l}\text { Número de } \\
\text { registros }\end{array}$ & Distribuição no Brasil & $\begin{array}{l}\text { Municípios com } \\
\text { registros }\end{array}$ & Fonte & Coleção \\
\hline \multicolumn{6}{|l|}{ BOTHRIURIDAE } \\
\hline Bothriurus araguayae Vellard, 1934 & 7 & $\begin{array}{l}\mathrm{BA}, \mathrm{DF}, \mathrm{GO}, \mathrm{MG}, \mathbf{M S} \\
\quad \text { PA, PI, RJ, SC, SP }\end{array}$ & $\begin{array}{c}2,8,9,11,15,18 \\
25,26\end{array}$ & $\mathrm{C}, \mathrm{E}$ & IBSP, MNRJ \\
\hline Bothriurus pora Mattoni \& Acosta, 2005 & 1 & MS & 19 & $\mathrm{C}, \mathrm{M}$ & IBSP \\
\hline $\begin{array}{l}\text { Brachistosternus simoneae Lourenço, } \\
2000\end{array}$ & 1 & $\mathrm{GO}, \mathbf{M S}$ & 25 & $\mathrm{~J}$ & IBSP \\
\hline $\begin{array}{l}\text { Brazilobothriurus pantanalensis } \\
\text { Lourenço \& Monod, } 2000\end{array}$ & 2 & MS & 8,9 & $\mathrm{C}, \mathrm{G}, \mathrm{K}$ & $\begin{array}{l}\text { IBSP, MHNG, } \\
\text { MNHN, MNRJ, } \\
\text { ZMH }\end{array}$ \\
\hline \multicolumn{6}{|l|}{ BUTHIDAE } \\
\hline Ananteris balzanii Thorell, 1891 & 7 & $\begin{array}{l}\mathrm{BA}, \mathrm{GO}, \mathrm{MG}, \mathbf{M S} \\
\text { MT, PA, SP }\end{array}$ & $1,4,6,9,16,25$ & $\mathrm{~B}, \mathrm{C}, \mathrm{I}, \mathrm{K}$ & IBSP, MZSP \\
\hline Ananteris mariaterezae Lourenço, 1987 & 1 & $\mathrm{TO}, \mathrm{MG}, \mathbf{M S}$ & 9 & $\mathrm{C}, \mathrm{I}, \mathrm{K}$ & $\mathrm{BMNH}$ \\
\hline Tityus bahiensis (Perty, 1833) & 10 & $\begin{array}{l}\text { ES, MG, MS, MT, RJ, } \\
\text { RS, GO, SC, SP, PR }\end{array}$ & $\begin{array}{l}1,5,7,8,13,16 \\
17,21,22,25\end{array}$ & $\mathrm{C}, \mathrm{K}$ & IBSP \\
\hline Tityus charreyroni Vellard, 1932 & 2 & $\mathrm{GO}, \mathbf{M S}, \mathrm{MT}$ & 8,16 & $\mathrm{C}$ & IBSP \\
\hline Tityus confluens Borelli, 1899 & 4 & MT, MS & $6,8,9,20,24$ & $\begin{array}{l}\mathrm{C}, \mathrm{O} \\
\mathrm{H}, \mathrm{A}\end{array}$ & IBSP \\
\hline $\begin{array}{l}\text { Tityus confluens bodoquena } \\
\text { Lourenço et al., 2004* }\end{array}$ & 1 & MS & 6 & $\mathrm{~L}$ & MNHN, CZUNB \\
\hline Tityus costatus (Karsch, 1879) & 1 & $\begin{array}{l}\text { BA, ES, MG, MS(?), } \\
\text { MT, PR, RJ, RS, SC, } \\
\text { SP }\end{array}$ & - & $\mathrm{C}$ & - \\
\hline Tityus indecisus Mello-Leitão, 1934* & 2 & MT, MS & 8,23 & $\mathrm{~F}$ & IBSP, MNRJ \\
\hline Tityus lutzi Giltay, 1928* & 1 & MT, MS & 1 & $\mathrm{D}$ & IBSP \\
\hline Tityus matogrossensis Borelli, 1901 & 7 & $\begin{array}{l}\text { BA, DF, GO, MG, MS, } \\
\text { MT, PI, SP, TO }\end{array}$ & $3,4,7,9,11,25$ & $\mathrm{C}$ & IBSP, MZSP \\
\hline Tityus paraguayensis Kraepelin, 1895 & 4 & $\begin{array}{c}\text { MS } \\
\text { BA, CE, DF, ES, GO, }\end{array}$ & $9,14,17,23$ & $\mathrm{C}, \mathrm{K}$ & IBSP, MZSP \\
\hline Tityus serrulatus Lutz \& Mello, 1922 & 1 & $\begin{array}{l}\text { MG, MS, PR, PE, RJ, } \\
\text { RN, SC, SE, SP, TO }\end{array}$ & 8 & $\mathrm{C}, \mathrm{P}$ & IBSP \\
\hline Tityus trivittatus Kraepelin, 1898 & 5 & MS, PR & $2,9,10,12,25$ & $\mathrm{C}, \mathrm{K}, \mathrm{N}$ & IBSP, FHC \\
\hline
\end{tabular}

*Táxons com distribuição ou status taxonômico incerto. Ver texto para maiores detalhes.

coletado no Mato Grosso do Sul foi encontrado nas coleções científicas examinadas.

O táxon que tem gerado maior discussão no que se refere ao aspecto sistemático e biogeográfico é $T$. confluens e suas duas subespécies T. confluens confluens e T. confluens bodoquena. A distribuição geográfica de $T$. confluens tem sido alvo de intensa discussão (LoURENÇO et al., 2004; BERTANi et al., 2005; LOURENÇO \& APARECIDA-DA-Silva, 2006, 2007), que resultou na publicação de outras espécies pertencentes ao complexo relacionado a Tityus confluens (ver LOURENÇO \& APARECIDA-DA-SilvA, 2006, 2007), além da subespécie $T$. confluens bodoquena, considerada válida e endêmica da região de Bonito, na Serra da Bodoquena, no estado do Mato Grosso do Sul (Lourenço \& ApArecidADA-SiLVA, 2006). No entanto, há consenso que T. confluens ocorre, pelo menos, na região do Pantanal matogrossense, nos estados do Mato Grosso e Mato Grosso do Sul, e no Chaco, na Argentina, Bolívia e Paraguai (Lourenço et al., 2004; LOURENÇO \& APARECIDA-DA-SILVA, 2006). Esta distribuição foi considerada e é apresentada na Tabela I. Esta questão poderia ser resolvida através de uma análise filogeográfica extensiva das espécies do complexo confluens, testando ainda a validade da subespécie $T$. confluens bodoquena.

Portanto, visto o exposto acima, consideramos que a lista de escorpiões do estado do Mato Grosso do Sul apresenta 13 espécies, excluindo-se aquelas que possuem inconsistências em termos taxonômicos, sistemáticos e/ ou biogeográficos. Tal riqueza pode ser considerada baixa quando comparada à de outros estados brasileiros como Amazonas ( 40 spp.), Bahia (28 spp.) e Pará ( 20 spp.), ou ainda quando comparado a localidades individuais, tais como a Reserva Florestal Adolfo Ducke, em Manaus, AM (8 spp.), a Floresta Nacional de Caxiuanã, em Melgaço e Portel, PA (6 spp.) e as regiões de Juruti (6 spp.) e Serra Norte/Carajás (6 spp.), ambas no Pará (HöFfer et al., 1996; PINTO-DARocha et al., 2002; 2007; PorTo et al., 2010a; BrazIL \& PORTO, 2011). Ainda assim, esta riqueza corresponde àquela apresentada por BRAZIL \& PORTO (2011) para o estado do 
Mato Grosso do Sul, colocando-o como, juntamente com o estado do Piauí, como o $8^{\circ}$ mais diverso do Brasil; além de representar cerca de metade da diversidade de escorpiões da região Centro-Oeste do Brasil (BRAZIL \& PORTO, 2011).

Principais grupos de pesquisa. Atualmente, a sistemática de escorpiões, especialmente das espécies que ocorrem no estado do Mato Grosso do Sul, constituem alvo esporádico de sistematas (brasileiros ou não) de aracnídeos. Desta forma, inexistem grupos de pesquisa que trabalhem exclusivamente com escorpiões desta região.

Principais acervos. A fauna de escorpiões do Mato Grosso do Sul encontra-se representada por relativamente poucos indivíduos em coleções zoológicas nacionais, destacando-se o MZSP e o IBSP, ambos localizados no estado de São Paulo. Todos os tipos dos táxons endêmicos do estado do Mato Grosso do Sul, e que não estão perdidos, encontram-se depositados nas referidas coleções ou em coleções internacionais, conforme listado na Tabela I.

Principais lacunas no conhecimento. Foram encontrados 60 registros não duplicados de escorpiões para o estado do Mato Grosso do Sul. Cada registro pode representar um ou mais lotes contendo um ou diversos escorpiões, coletados em um mesmo município. Devido à imprecisão de algumas localidades, por se tratar de registros muito antigos (p. ex., BorelLI, 1901), para o cálculo do número de registros, as informações sobre estas localidades foram suprimidas. Apenas 26 dos 79 municípios (32,9\%) do estado do Mato Grosso do Sul apresentaram pelo menos um registro de escorpião, exibindo um padrão heterogêneo do conhecimento da região, como também encontrado para escorpiões do estado da Bahia (PorTo et al., 2010b).

A espécie com o maior número de registros foi $T$. bahiensis, com dez registros, seguida por $B$. araguayae $(\mathrm{n}=8)$, A. balzanii $(\mathrm{n}=7)$ e $T$. confluens $(\mathrm{n}=5)$. A. mariaterezae, $B$. pora, B. simoneae, T. confluens bodoquena e T. serrulatus só possuem um registro para o estado do Mato Grosso do Sul. Este número reduzido de registros também foi encontrado para aranhas do estado de São Paulo (BRESCOVIT et al., 2011)

A distribuição dos registros pelos biomas do estado também é heterogênea. As áreas cobertas pelos domínios da Mata Atlântica e do Pantanal apresentam poucos registros de escorpiões, havendo maior amostragem na região dos domínios do Cerrado (Fig. 2). Este padrão é semelhante ao apresentado por BRESCOVIT et al. (2011) para aranhas do Mato Grosso do Sul, e pode refletir questões históricas de amostragem, tais como a facilidade de acesso, além da proximidade com a capital do estado, Campo Grande.

As informações aqui apresentadas mostram que o estado do Mato Grosso do Sul contribui significativamente para a diversidade de escorpiões do Brasil, apresentando

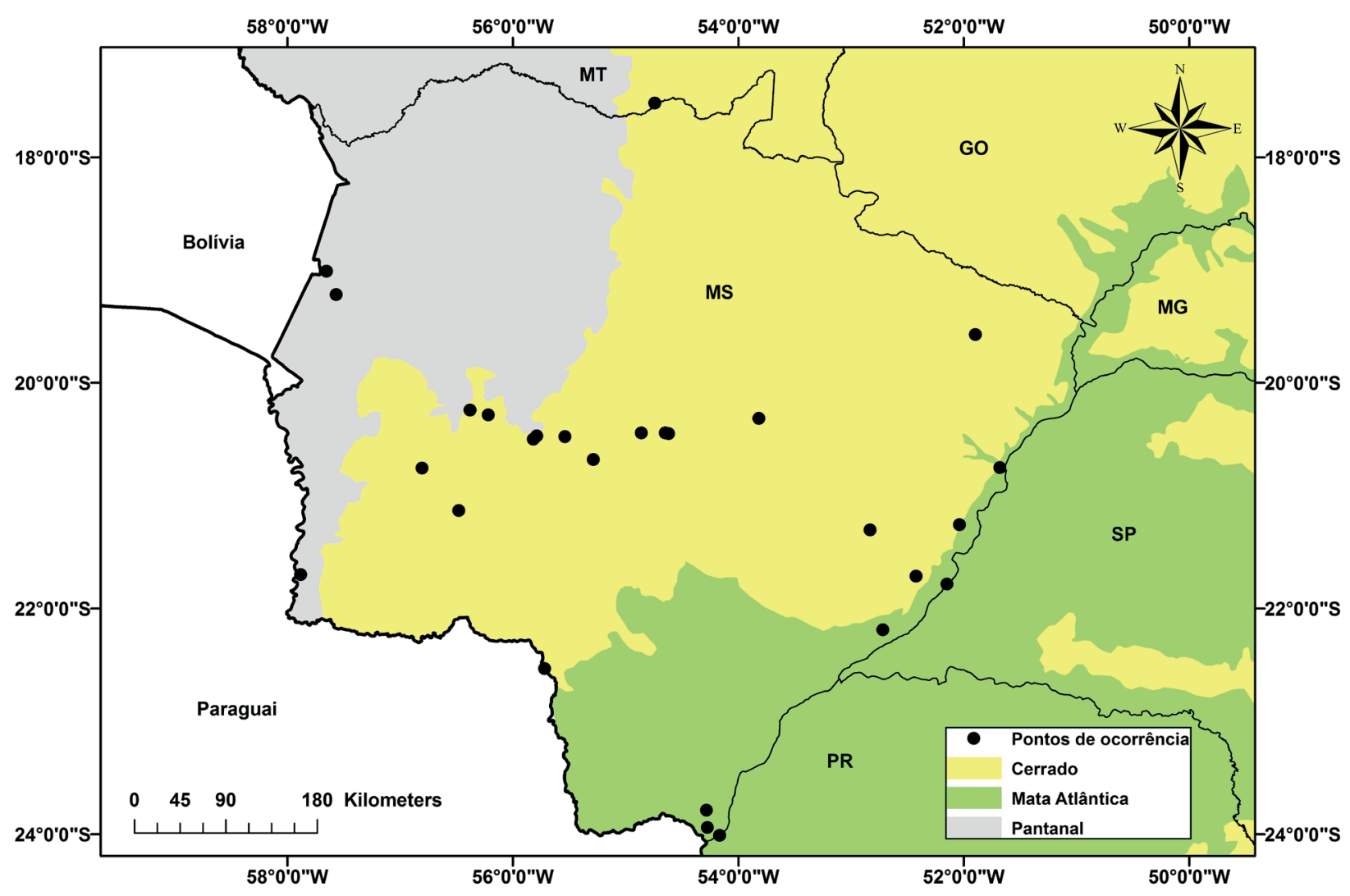

Fig. 2. Pontos de ocorrência de espécies de Scorpiones no estado do Mato Grosso do Sul. Cada ponto representa pelo menos uma ocorrência de pelo menos uma espécie de escorpião (GO, Goiás; MG, Minas Gerais; MS, Mato Grosso do Sul; MT, Mato Grosso; PR, Paraná; SP, São Paulo). 
diversos elementos endêmicos, tais como $\mathrm{Bo}$. pora e $\mathrm{Br}$. pantanalensis, ou espécies com distribuição restrita no Brasil, tais como T. charreyroni e Bo. simoneae. No entanto, é preciso melhorar a representatividade da escorpiofauna local em coleções científicas, através da amostragem de áreas visivelmente subamostradas, tais como aquelas dominadas pela Mata Atlântica e o Pantanal. Este último domínio, o Pantanal, possui características ecológicas únicas no país, sendo importante o desenvolvimento de estudos ecológicos e biogeográficos que permitam entender a evolução deste ambiente com os processos de diversificação e a manutenção da fauna de escorpiões na região. Isto poderá ajudar, por exemplo, a solucionar as questões envolvendo as espécies de escorpiões do complexo confluens.

Perspectivas de pesquisa para o grupo nos próximos 10 anos. O estudo da escorpiofauna do estado do Mato Grosso do Sul, potencialmente, pode seguir pelo menos três vertentes nos próximos anos: (1) avaliação da validade de táxons historicamente problemáticos (ex.: $T$. indecisus, T. confluens bodoquena, T. lutzi), que pode ser feito através do exame de material-tipo destas espécies e ainda com análises filogeográficas e/ou filogenéticas; (2) melhoria do conhecimento da distribuição geográfica das espécies existentes e da diversidade local nos diversos ecossistemas da região; (3) estudos de ecologia de populações, associando as espécies presentes a variações em características abióticas dos ecossistemas onde vivem, semelhante ao que tem sido apresentado para escorpiões de outras regiões do Brasil (ex.: HÖFFER et al., 1996; DiAs et al., 2006; ARAújo et al., 2010; LIRA et al., 2013).

Agradecimentos. A Fundação de Apoio ao Desenvolvimento do Ensino, Ciências e Tecnologia do Estado de Mato Grosso do Sul (Fundect) e a Superintendência de Ciências e Tecnologia do Estado de Mato Grosso do Sul (Sucitec/MS) pelo convite de participação neste fascículo especial da Iheringia, série Zoologia e o suporte financeiro para sua publicação. Somos gratos à Ricardo Pinto da Rocha pelo envio de informações referentes à coleção de escorpiões do MZSP, à Marielle Schneider por sugestões no manuscrito e à Viviane Mattos, Rafael Indicatti e Douglas Araújo pelas fotos.

\section{REFERÊNCIAS BIBLIOGRÁFICAS}

Albuquerque, C. M. R.; Porto, T. J.; Amorim, M. L. P. \& Santana-Neto, P. L. 2009. Escorpionismo por Tityus pusillus Pocock, 1893 (Scorpiones, Buthidae) no Estado de Pernambuco. Revista da Sociedade Brasileira de Medicina Tropical 42:206-208.

Álvares, E. S. S.; De Maria, M.; Amancio, F. F. \& Campolina, D. 2006. Primeiro registro de escorpionismo causado por Tityus adrianoi Lourenço (Scorpiones: Buthidae). Revista da Sociedade Brasileira de Medicina Tropical 39:383-384.

Araújo, C. S.; Candido, D. M.; Araújo, H. F. P.; Dias, S. C. \& VASCONCELLOS, A. 2010. Seasonal variations in scorpion activities (Arachnida: Scorpiones) in an area of Caatinga vegetation in northeastern Brazil. Zoologia 27:372-376.

Bertani, R.; Martins, R. \& Carvalho, M. A. 2005. Notes on Tityus confluens Borelli, 1899 (Scorpiones: Buthidae) in Brazil. Zootaxa 869:1-7.

BoRelli, A. 1901. Scorpioni raccolti dal Dott. Filippo Silvestri nella Repubblica Argentina e regioni vicine. Bollettino dei Musei di Zoologia ed Anatomia Comparata della Reale Universitá di Torino 16(403):1-12.

Brandão, R. A. \& Françoso, R. D. 2010. Acidente por Rhopalurus agamemnon (Koch, 1839) (Scorpiones, Buthidae). Revista da Sociedade Brasileira de Medicina Tropical 43:342-344.
Brazil, T. K. \& Porto, T. J. 2011. Os escorpiões. Salvador, Edufba. vol. $1,84 \mathrm{p}$.

Brescovit, A. D.; Oliveira, U. \& Santos, A. J. 2011. Aranhas (Araneae, Arachnida) do Estado de São Paulo, Brasil: diversidade, esforço amostral e estado do conhecimento. Biota Neotropica 11:1-31.

BÜCHERL, W. 1959. Escorpiões e Escorpionismo no Brasil. 10. Catálogo da coleção escorpiônica do Instituto Butantan. Memórias do Insituto Butantan 29:255-275.

BÜCHERL, W. 1960-62. Escorpiões e escorpionismo no Brasil. XI. Revisão dos Bothriurideos da coleção escorpiônica do Museu Nacional do Rio de Janeiro. Memórias do Insituto Butantan 30:187-206.

Candido, D. M. 1999. Escorpiões. In: Brandẽo, C. R. F. \& Cancello, E. M. org. Biodiversidade do Estado de São Paulo. São Paulo, Fapesp. vol. 5, p. 23-34.

CaRvalho, L. S.; Santos, M. P. D. \& Dias, S. C. 2007. Escorpionismo na zona rural de Teresina, Estado do Piauí: relato de casos de envenenamento. Revista da Sociedade Brasileira de Medicina Tropical 40:491.

Dias, S. C.; Candido, D. M. \& Brescovit, A. D. 2006. Scorpions from Mata do Buraquinho, João Pessoa, Paraíba, Brazil, with ecological notes on a population of Ananteris mauryi Lourenço (Scorpiones, Buthidae). Revista Brasileira de Zoologia 23:707-710.

Fet, V.; Sissom, S. D.; Lowe, G. \& Braunwalder, M. E. 2000. Catalog of the Scorpions of the World (1758-1998). New York, New York Entomological Society. 690p.

Höffer, H.; Wollsscheid, E. \& Gasnier, T. R. 1996. The Relative abundance of Brotheas amazonicus (Chactidae, Scorpiones) in different habitat types of a Central Amazon Rainforest. Journal of Arachnology 24:34-38.

Giupponi, A. P. L.; Vasconcelos, E. G. \& Lourenço, W. R. 2009. The genus Ananteris Thorell, 1891 (Scorpiones, Buthidae) in southeast Brazil, with the description of three new species. ZooKeys 13:29-41.

KurY, A. B. \& Nogueira, A. C. 1999. Annotated check list of type specimens of Arachnida in the Museu Nacional, Universidade Federal do Rio de Janeiro, I. Scorpiones, Pseudoscorpiones and Solifugae. Publicações Avulsas do Museu Nacional 77:1-19.

Lira-Da-Silva, R. M.; Amorim, A. M.; Carvalho, F. M. \& Brazil, T. K. 2009. Acidentes por escorpião na cidade do Salvador, Bahia, Brasil (1982 - 2000). Gazeta Médica da Bahia 79:43-49.

Lira, A. F. A.; Souza, A. M.; Silva Filho, A. A. C. \& Albuquerque, C. M. R. 2013. Spatio-temporal microhabitat use by two co-occurring species of scorpions in Atlantic rainforest in Brazil. Zoology 116:182- 185.

LOURENÇO W. R. 1979. Contribution â la connaissance du scorpion Buthidae Tityus mattogrossensis Borelli, 1901 (Morphologie, écologie, biologie et développement postembryonnaire). Bulletin du Muséum National d'Histoire Naturelle 4(1A):95-117.

LOURENÇO W. R. 1982. Revision du genre Ananteris Thorell, 1891 (Scorpiones, Buthidae) et description de six especes nouvelles. Bulletin du Muséum National d'Histoire Naturelle 4(1/2):119-151.

LOURENÇO W. R. 2000. Confirmation de la présence du genre Brachistosternus Pocock (Scorpiones, Bothriuridae) au Brésil et description d'une espèce nouvelle. Revue Arachnologique 13(6):93-100.

LOURENÇO W. R. 2002. SCORPIONS of Brazil. Paris, Les Éditions l'If. 308p.

LourençO, W. R. \& ApareCIDA-DA-Silva, E. 2006. A reappraisal of the geographical distribution of the complex Tityus confluens Borelli, 1899 (Scorpiones: Buthidae) with the description of a new species. Entomologische Mitteilungen aus dem Zoologischen Museum Hamburg 14(174):307-320.

Lourenç̧, W. R. \& APARECIDA-DA-Silva, E. 2007. New evidence for a disrupted distribution pattern of the 'Tityus confluens' complex, with the description of a new species from the State of Pará, Brazil (Scorpiones, Buthidae). Amazoniana 19(3/4):77-86.

Lourenço W. R.; Cabral, B. C. \& Bruehmuller Ramos, E. C. 2004. Confirmation of Tityus confluens Borelli, 1899 (Scorpiones, Buthidae) in Brazil and description of a new subspecies from the State of Mato Grosso do Sul. Boletín de la Sociedad Entomológica Aragonesa 34:27-30.

LOURENÇO, W. R. \& EICKSTEDT, V. R. 2009. Escorpiões de importância médica. In: Cardoso, J. L. C.; França, F. D. S.; Wen, F. H.; Malaque, C. M. S. \& HAdDAD JR., V. org. Animais peçonhentos no Brasil: biologia, clínica e terapêutica dos acidentes. São Paulo, Sarvier, p. $198-213$.

LOURENÇO, W. R. \& Monod, L. 2000. Description of a new genus and species of scorpion (Bothriuridae) from Brazil. Ekológia (Bratislava) 19(3): 145-152. 
Mattoni, C. I. \& Acosta, L. E. 2005. A new species of Bothriurus from Brazil (Scorpiones, Bothriuridae). Journal of Arachnology 33:735-744

Maury, E. A. 1970. Redescripción y distribución en la Argentina de Tityus trivittatus trivittatus Kraepelin, 1898 (Scorpiones, Buthidae). Comentarios sobre sus hábitos domiciliarios y su peligrosidad. Physis 29:405-421.

MaURY, E. A. 1974. Escorpiofauna Chaqueña. II. Tityus confluens Borelli 1899 (Buthidae). Physis (C) 33(86):85-92.

Maury, E. A. 1997. Tityus trivittatus en la Argentina. Nuevos datos sobre distribución, partenogenesis, sinantropía y peligrosidad (Scorpiones, Buthidae). Revista del Museo Argentino de Ciencias Naturales "Bernardino Rivadavia" 24:1-24.

Pinto-Da-Rocha, R.; AraúJo, C.; Barreiros, J. A. P. \& Bonaldo, A. 2007. Arthropoda, Arachnida, Scorpiones: Estação Científica Ferreira Penna and Juruti Plateau, Pará, Brazil. Check List 3:145-147.

Pinto-da-Rocha, R.; Gasnier, T. R.; Brescovit, A. D. \& ApolináRio, F. B. 2002. Broteochactas fei, a new scorpion species from Brazilian Amazônia, with notes on its abundance and association with termites. Revista Ibérica de Aracnología 6:195-202.

Porto, T. J.; Caldas, E. A.; Cova, B. O. \& Santos, V. M. N. 2010a. Primeiro relato de acidentes escorpiônicos causados por Tityus martinpaechi Lourenço, 2001 (Scorpiones, Buthidae). Revista de Ciências Médicas e Biológicas 9:266-269.

Porto, T. J.; BraziL, T. K. \& Lira-DA-SiLVA, R. M. 2010b. Scorpions, state of Bahia, northeastern Brazil. Check List 6:292-297.

Souza, C. A. R.; Candido, D. M.; LuCas, S. M. \& Brescovit, A. D. 2009. On the Tityus stigmurus complex (Scorpiones, Buthidae). Zootaxa 1987:1-38. 